\title{
Semiquantitative analyses of fine needle aspirates from benign and malignant breast lesions
}

\author{
J ST J THOMAS, * E A MALLON, * W D GEORGE $\dagger$ \\ From the Departments of * Pathology and $\nmid$ Surgery, Western Infirmary, Glasgow, Scotland
}

SUMMARY A scoring system for the assessment of fine needle aspirates of benign and malignant breast lesions was devised which showed a positive correlation $(r=0.67)$ between the scores obtained from the fine needle aspirates from ductal carcinomas and the Bloom and Richardson-type scores for their paired excision biopsy specimens. This system permitted grades II and III ductal breast carcinoma to be distinguished reliably from grade I tumours but no correlation with the lymph node state of patients with breast carcinoma was shown. Some overlap between the scores for grade I ductal carcinomas and some benign lesions was found, and this underlines a need for caution in the reporting of such equivocal aspirates. No cytological features that distinguished reliably ductal from lobular carcinoma were identified but the same spectrum of severity of cytological abnormality in the ductal and lobular carcinoma aspirates was seen.

This system may be of prognostic value in the assessment of lobular carcinoma which has hitherto defied histological grading.

With the advent of a National Breast Cancer Screening Programme the requirement for preoperative diagnosis of breast disease will increase considerably and will result in a growing demand for Tru-Cut biopsy and fine needle aspiration cytology. Indeed, in some centres the use of intraoperative frozen section diagnosis for breast disease has almost disappeared (C W Elston, personal communication). We have extensive experience of fine needle aspiration cytology of breast disease, deriving from a weekly specialist breast clinic where this diagnostic approach is performed routinely. To assess objectively the uses and limitations of this technique we devised a scoring system for the analysis of breast aspirates and applied this to aspirates from histologically confirmed benign and malignant breast diseases. Specifically, we set out to determine to what extent histological grade can be predicted by preoperative fine needle aspiration, whether there are measurable features that allow benign breast diseases to be distinguished reliably from carcinomas, and whether ductal carcinoma can be distinguished from lobular carcinoma cytologically.

\section{Material and methods}

Fine needle aspirates that were adequate for diagnos-

Accepted for publication 21 July 1988 tic purposes were identified from the files of the department of pathology where follow up biopsy confirmation of the diagnosis was available. The fine needle aspirates were obtained by the surgical staff attending the breast clinic using a standard technique. The aspirates were then smeared by the pathologist in a side room of the clinic and after air drying the smears were stained using the Diff Quik technique. Fine needle aspirates from a total of 42 cases of ductal carcinoma, 16 cases of lobular carcinoma, 11 cases of fibroadenoma, and 10 cases of other benign breast disease were reviewed. The histology of all cases was reviewed and the histology from the ductal carcinomas was scored and graded according to modified Bloom and Richardson' criteria outlined briefly below. Fine needle aspirates were subjected to the following scoring system without knowledge of the final histological diagnosis of the subsequently excised lesion. To provide reasonable numbers of lobular carcinomas for comparison with the ductal carcinomas a relative excess of the former type of neoplasm was deliberately sought. The distribution of cases in this series is not therefore representative of the distribution of diagnoses of breast disease in this hospital.

\section{MEASUREMENTS}

Breast fine needle aspirates

The smears were assessed for:

(1) Cell to cell cohesion: Cells tightly cohesive with only 
slight loss of clustering (score 1); clustering was the predominant feature of the smear but areas of loss of cell to cell cohesion easily recognised (score 2 ); clustering, although present, was not a striking feature of the smear (score 3).

(2) Nuclear size: Maximum nuclear diameter was measured using an eye piece graticule. Ten high power fields $(\times 40)$ were assessed and 10 cells in each field measured. Cells were not measured where cellular overlap obscured nuclear boundaries or where drying artifact was evident. Scoring was as follows: average maximum nuclear diameter $<13 \mu \mathrm{m}$, score 1 ; average maximum nuclear diameter 13-15 $\mu \mathrm{m}$, score 2; average maximum nuclear diameter $>15 \mu \mathrm{m}$, score 3 . (3) Nuclear pleomorphism: Mild, score 1; moderate, score 2; severe, score 3.

(4) Nucleolar prominence: Nucleoli inconspicuous, (score 0); nucleoli enlarged and conspicuous (score 1).

\section{MEASUREMENTS}

Biopsy specimens

(1) Ductal carcinomas: All sections were assessed and evaluated according to Bloom and Richardson criteria, ${ }^{1}$ which we have modified slightly. Both the scores and the grades were noted for each case.

Our minor modification of the Bloom and Richardson criteria is outlined in table 1.

(2) Lobular carcinomas: Histological confirmation alone.

(3) Benign conditions including fibroadenomas: Histological confirmation alone.

Cytological scores for all fine needle aspirates were compared with the histological classification of the lesion concerned (fig 1). The scores from the fine needle aspirates from the ductal carcinomas were also compared using the modified Bloom and Richardson scores for their paired histological specimens (fig 2). The benign lesions were separated into two histological groups: (i) fibroadenomas; and (ii) other benign breast diseases.

\section{Results}

Many of the fibroadenomas had a characteristic

Table 1 Modified Bloom and Richardson scoring system for histological sections from ductal breast carcinoma

\begin{tabular}{llll}
\hline \multicolumn{3}{c}{ Histological feature } & \\
\cline { 2 - 4 } Score & $\begin{array}{l}\text { Tubule } \\
\text { formation }\end{array}$ & Nuclear appearance & Mitotic rate \\
\hline 1 & Large areas & $\begin{array}{l}\text { Small, uniform, } \\
\text { vesicular } \\
\text { Large, nucleoli seen, } \\
\text { moderate pleomorphism } \\
\text { Pleomorphic, bizarre }\end{array}$ & $\begin{array}{c}\leqslant 2 / \mathrm{HPF} \\
\text { powered field }\end{array}$ \\
3 & Some areas & $\geqslant 3 / \mathrm{HPF}$ \\
\hline
\end{tabular}

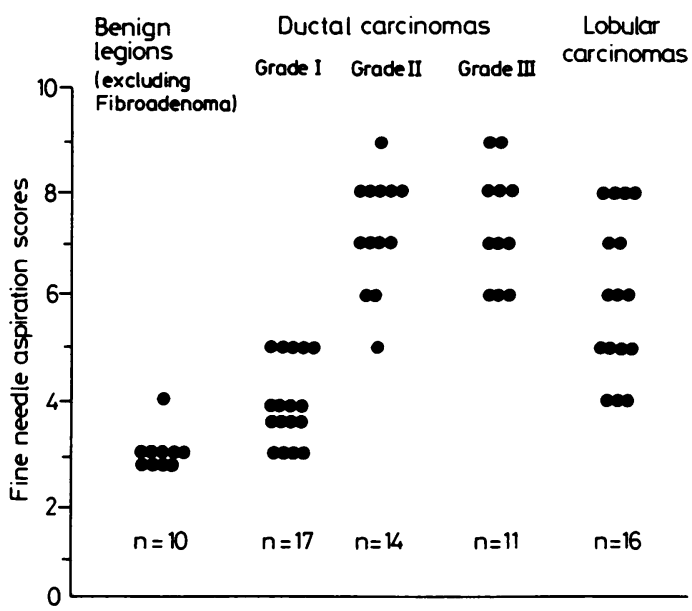

Fig 1 Comparison of fine needle aspiration scores for benign breast diseases (excluding fibroadenoma), modified Bloom and Richardson grades for ductal carcinomas, and for lobular carcinomas.

cytological pattern of tight clusters of small epithelial cells in addition to a separate population of intervening "naked nuclei". All smears showing such a pattern analysing nuclear size and pleomorphism for the clusters and "naked nuclei" were measured separately. The cell clusters showed an average maximum nuclear diameter of $11 \mu \mathrm{m}$ (range: $10 \mu \mathrm{m}$ to $12 \mu \mathrm{m}$ ) while the naked nuclei showed an average maximum nuclear diameter of $13 \mu \mathrm{m}$ (range $12 \mu \mathrm{m}$ to $14 \mu \mathrm{m}$ ). These smears also showed a greater degree of nuclear pleomorphism than in smears taken from other types of benign lesions. In view of the fact that the assessment of the fibroadenoma smears entailed the application of all or part of our scoring system twice to the

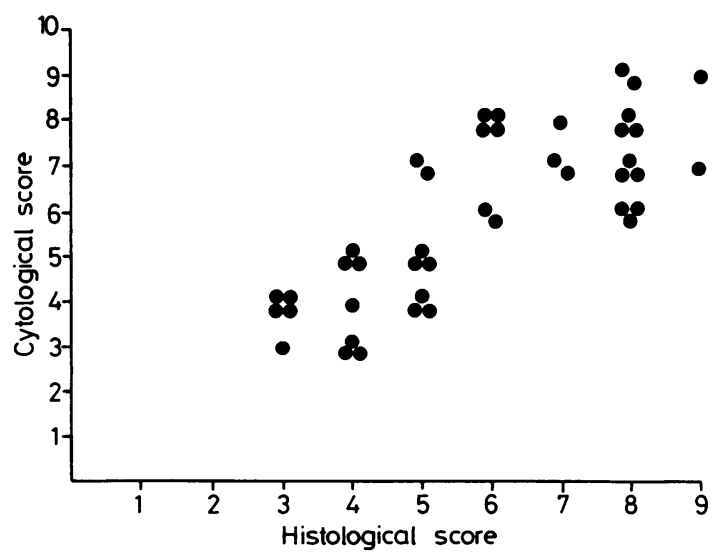

Fig 2 Ductal carcinomas: comparison of fine needle aspiration scores with modified Bloom and Richardson scores for their paired excision biopsy specimens. 
Table 2 Mean values for cytological scores for their corresponding histological lesions and their $95 \%$ confidence limits

\begin{tabular}{|c|c|c|c|}
\hline $\begin{array}{l}\text { Histological } \\
\text { lesion }\end{array}$ & $\begin{array}{l}\text { Mean } \\
\text { cytological } \\
\text { score }\end{array}$ & $\begin{array}{l}\text { Standard } \\
\text { deviation }\end{array}$ & $\begin{array}{l}95 \% \\
\text { confidence } \\
\text { limits }\end{array}$ \\
\hline $\begin{array}{l}\text { Benign lesions } \\
\text { (excluding fibroadenomas) } \\
\text { Ductal carcinomas: }\end{array}$ & $3 \cdot 1$ & $0 \cdot 1$ & $3 \cdot 04-3 \cdot 16$ \\
\hline $\begin{array}{l}\text { All grades } \\
\text { Grade I } \\
\text { Grade II } \\
\text { Grade III } \\
\text { Lobular carcinomas }\end{array}$ & $\begin{array}{l}5 \cdot 97 \\
4 \cdot 05 \\
7 \cdot 21 \\
7 \cdot 36 \\
6 \cdot 0\end{array}$ & $\begin{array}{l}3 \cdot 24 \\
0 \cdot 56 \\
1 \cdot 10 \\
1 \cdot 25 \\
2 \cdot 53\end{array}$ & $\begin{array}{l}4 \cdot 97-6 \cdot 97 \\
3 \cdot 77-4 \cdot 33 \\
5 \cdot 01-9 \cdot 41 \\
4 \cdot 86-9 \cdot 86 \\
4 \cdot 74-7 \cdot 26\end{array}$ \\
\hline
\end{tabular}

same smear (firstly to the clusters and then to the intervening naked nuclei) we did not feel that we could then make a valid comparison of the scores obtained with other benign and malignant lesions where the scoring system could be applied once to the whole smear. Our measurements included aspirates from 10 cases of other benign breast diseases, all of which were cases showing a wide spectrum of lesions seen in fibrocystic change. Only one of these cases showed more than the minimum score and this case scored four points. Large numbers of apocrine-type epithelial cells were present in the smears from this case and apocrine metaplasia was a prominent component of the fibrocystic change seen in the histological sections.

The mean values for the cytological scores and their
95\% confidence limits are given in table 2 .

Statistical analysis of the results for the ductal carcinomas using Student's $t$ test and Snedecor's $F$ test showed highly significant differences between the cytological scores for grade I carcinomas and grades II and III carcinomas (I $v$ II $t=9.375$; I $v$ III $t=8.46$; I $v$ II + III $t=58$ ), but no significant difference between grades II and III. There was no significant difference between the scores obtained for the grade I carcinomas and the benign lesions. Twelve of the 17 grade I carcinomas showed scores of 3 or 4 , reflecting the cytological blandness of this group of tumours (fig 3 ).

We have reviewed the smears from these low grade carcinomas in an attempt to determine those features present which permit their distinction from benign lesions. In retrospect, a large number of these low grade malignant smears showed an overall cellularity that was considerably greater than that seen in the benign lesion smears; a feature that was not included in our scoring system. We also noted subtle degrees of nuclear pleomorphism and loss of cell to cell cohesion that would have been impossible to score using our comparatively crude criteria. Typical appearances seen in smears from middle and high grade ductal carcinomas are shown in figs 4 and 5 , respectively. Correlative analysis of the comparison between the fine needle aspiration scores for the ductal carcinoma aspirates and the modified Bloom and Richardson scores for their paired histology showed a significant positive correlation (correlation coefficient, $r=0.67$ ).

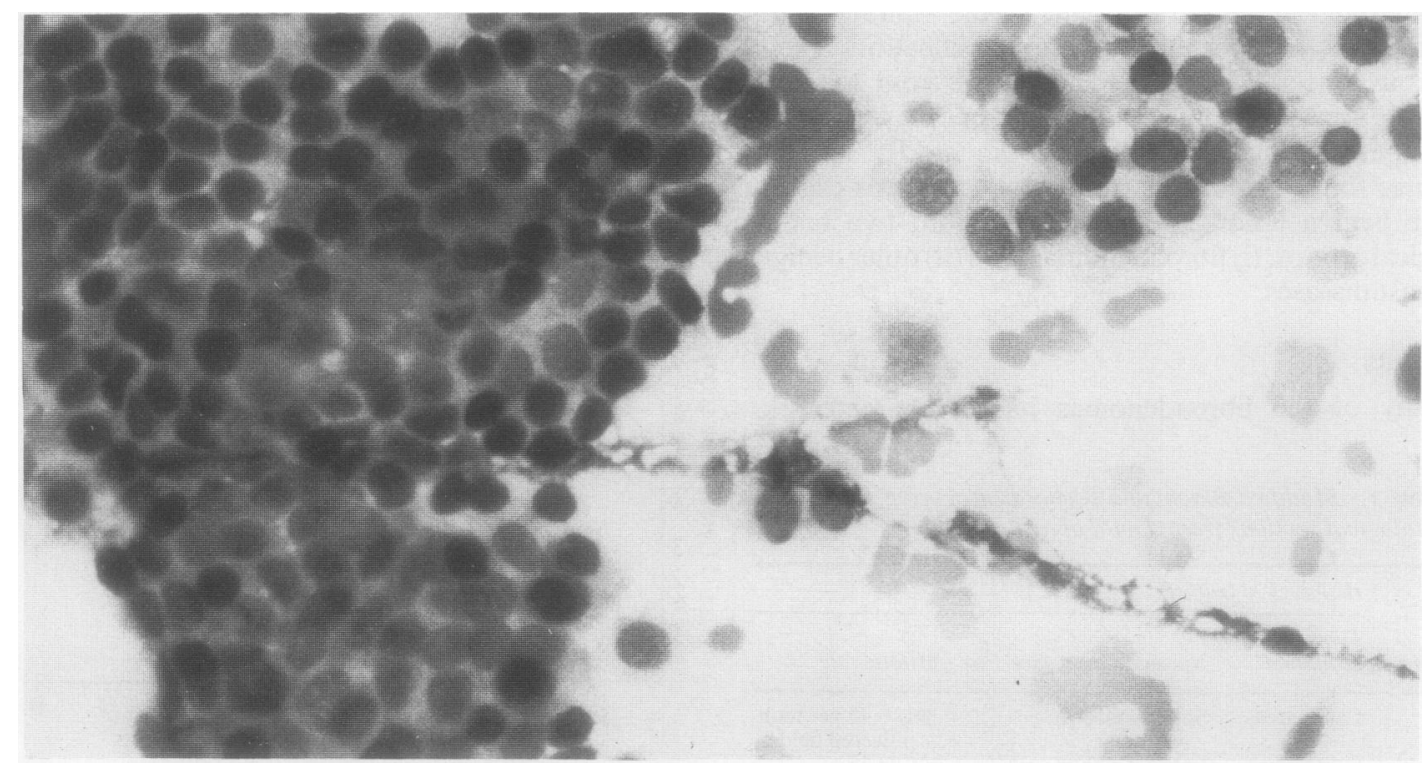

Fig 3 Low grade ductal carcinoma fine needle aspiration: note prominent clustering, comparatively small nuclear size, mild nuclear pleomorphism and lack of nucleolar prominence. Scores: clustering 1; nuclear size (11 um) 1; pleomorphism 1; nucleoli 0. 


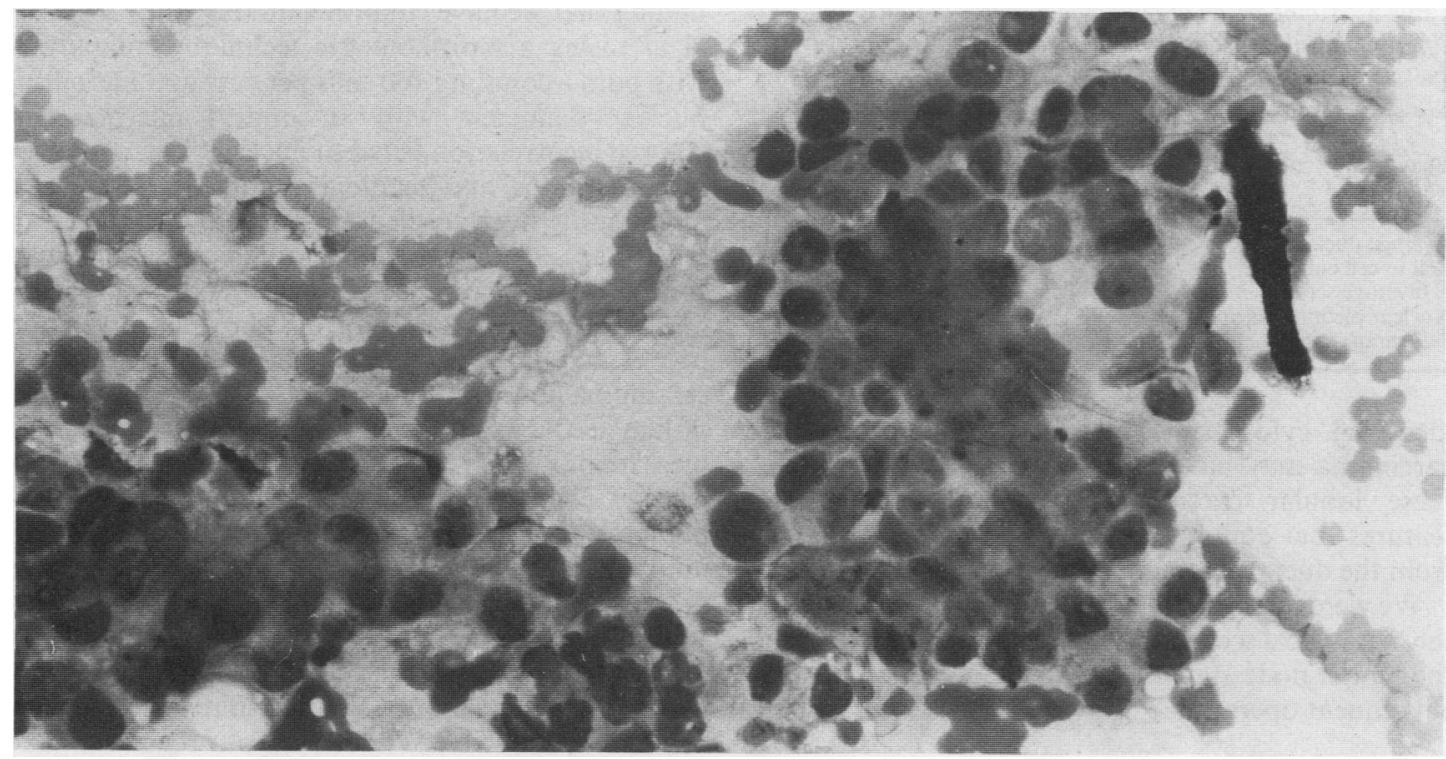

Fig 4 Middle grade ductal carcinoma fine needle aspiration: clustering remains dominant overall feature. Nuclear pleomorphism is of a moderate degree and nucleoli are enlarged and easily identified. Scores: clustering 1; nuclear size (14 $\mu m$ ) 2; pleomorphism 2; nucleoli 1.

Comparisons of the scores obtained for the separate variables assessed in the smears obtained from the ductal carcinomas are shown in table 3.

The distribution of cases among the three histological grades showing nucleolar prominence was as follows: grade I, five of 17 cases; grade II, seven of 14 cases; grade III, five of 11 cases.

Cytological scoring of the fine needle aspirates from the lobular carcinomas showed the same range of scores as seen in the ductal carcinomas (fig 2). Two of the 16 lobular carcinomas showed cytoplasmic vacuolation, and in one of these a mucicarmine stain

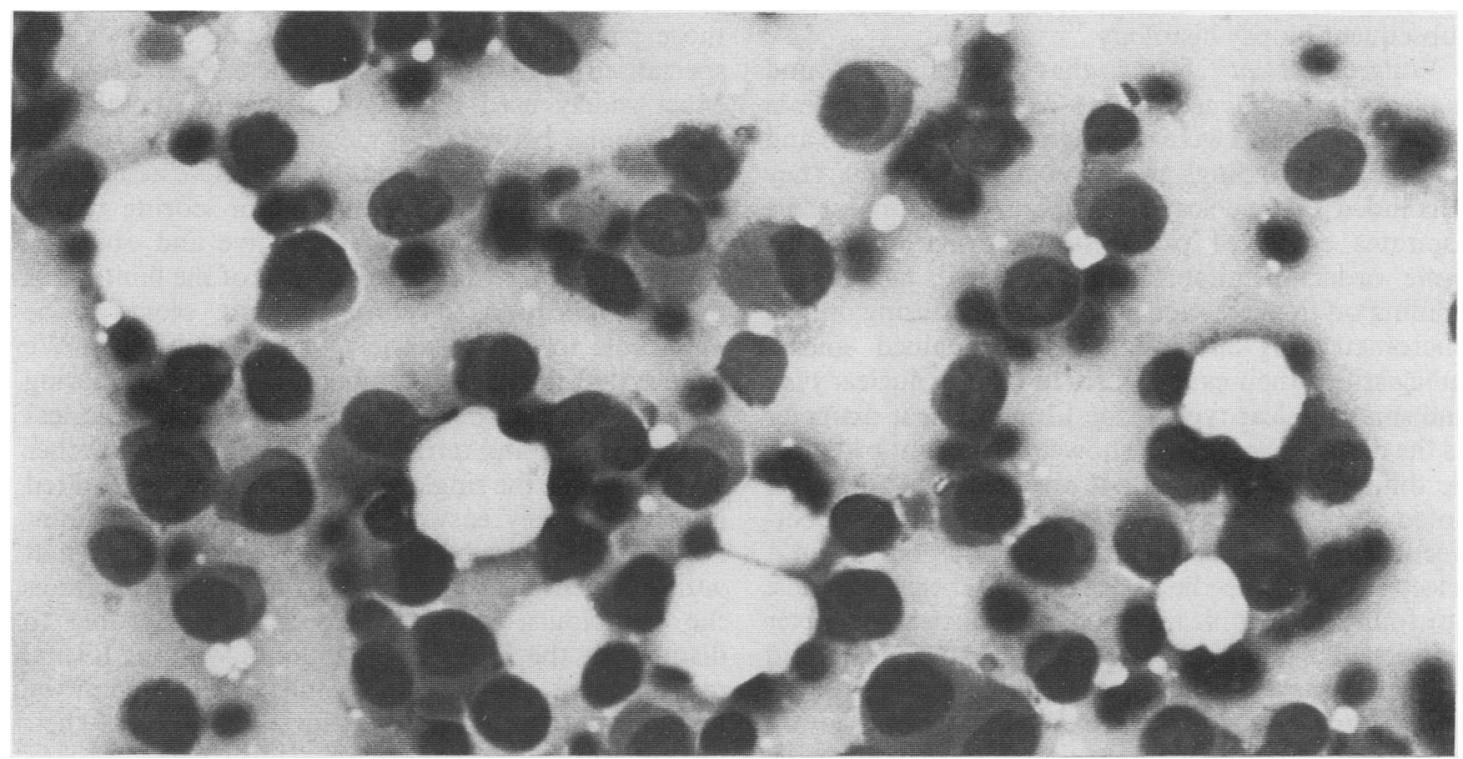

Fig 5 High grade ductal carcinoma fine needle aspiration: there is complete loss of clustering of cells and the nuclei are large and pleomorphic. Scores: clustering 3; nuclear size (17 $\mu \mathrm{m})$ 3; pleomorphism 3; nucleoli 0. 
Table 3 Comparison of cytological scores obtained for different variables measured on smears for each grade of ductal carcinoma

\begin{tabular}{llll}
\hline \multirow{2}{*}{$\begin{array}{l}\text { Cytological } \\
\text { feature }\end{array}$} & \multicolumn{3}{l}{ Histological grade } \\
\cline { 2 - 4 } & $I$ & $I I$ & $I I I$ \\
\hline $\begin{array}{l}\text { Average nuclear size } \\
\text { Cell to cell cohesion } \\
\begin{array}{c}\text { (average score) } \\
\text { Nuclear pleomorphism } \\
\text { (average score) }\end{array}\end{array}$ & $12.3 \mu \mathrm{m}$ & $14.1 \mu \mathrm{m}$ & $15.7 \mu \mathrm{m}$ \\
& 1.3 & 1.7 & 2.0 \\
\hline
\end{tabular}

identified cytoplasmic mucin, suggesting that the carcinoma may have been lobular in type. Most of these lobular carcinomas, therefore, showed no features that could be relied on to distinguish them from the ductal carcinomas.

We were unable to identify any correlation between the cytological scores obtained for the carcinomas and the lymph node state of these patients determined at subsequent operation.

\section{Discussion}

In the hands of experienced pathologists the Bloom and Richardson grading system or more formal histomorphometric analysis ${ }^{2}$ for ductal breast carcinomas has provided valuable prognostic information. Although previous studies have shown that morphometric analysis of fine needle aspirates from breast carcinomas is of value in the estimation of prognosis, ${ }^{3-7}$ there has been little work on the formal correlation of fine needle aspiration findings with subsequent biopsy histology.

Walgren et $a^{3}$ found that nuclear area and nucleolar prominence in fine needle aspirates from breast carcinomas were of prognostic importance and related their findings to follow up histology. They concluded that nuclear size measured on fine needle aspirates conveyed prognostic information of the same order of importance as formal histological grading on tissue sections. Zajdela et al, ${ }^{4}$ using optical micrometry on air dried, Giemsa stained smears subclassified their cancer cases into large nuclear type and small nuclear type, using $12 \mu \mathrm{m}$ nuclear diameter as the dividing point, and showed significant prognostic differences between these two groups. They also noted a significant overlap of nuclear diameter between their small nuclear diameter carcinomas and a selection of fine needle aspirates from benign lesions, but found no significant overlap of nuclear diameter between the small nuclear diameter carcinomas and the large nuclear diameter carcinomas. KuenenBoumeester et $a^{5}$ failed to show any association between their morphometric variables of fine needle aspirates and their patients' lymph node state, a finding that we have confirmed in this study. Mossler et $a l^{6}$ using a morphometric technique comparable with ours - counting 100 cells per case and measuring nuclear diameters with an optical micrometershowed a correlation between nuclear area, oestrogen receptor status, and histological grade. In an attempt to quantify nuclear pleomorphism the variance of nuclear area in fine needle aspirates has been shown to correlate with recurrence of tumour and survival. ${ }^{57}$

A recently reported study ${ }^{8}$ has shown a correlation between crude survival figures for all types of breast carcinoma and a scoring system applied to a mixture of fine needle aspirates and touch preparations from these lesions. This scoring system included the evaluation of cellular clustering, cell size, nuclear morphology and staining, and nucleolar staining and mitotic activity. Formal measurements of nuclear size do not seem to have been taken into account in this study. We examined this system and found it difficult to apply to our cases and therefore devised a scoring system that included a semiquantitative element to make it reproducible. We devised our regime to take account of those features which have been shown in previous studies $^{3-8}$ to be of prognostic importance and which we have found easy to assess. It is accepted that all grading systems, be they for cytological or histological preparations, prove difficult to reproduce both from an intra- and interobserver point of view, principally due to the subjective components inherent in such systems. Unfortunately, to make any system more objective and therefore more reproducible, quantitative analysis becomes imperative and this inevitably restricts the application of the system to those pathologists who have the time and possibly specialised equipment to make these measurements. Most widely used grading systems are therefore a compromise between the ideal of a totally objective measured set of criteria and the subjective assessment of a variety of different features. Our scoring system also includes a mixture of subjective and objective components but, with the exception of the limitations of its application to fibroadenomas, seems to be applicable to a wide variety of breast diseases. We believe that the subjective components of the scoring system are simple to assess and, we would hope, be less prone to inter- and intraobserver variation than other systems, while the single measurement that is required is comparatively easy to perform, requiring a minimum of specialised equipment. We would hope that with sufficient exposure to breast fine needle aspirates the pathologist will learn to at least be able to distinguish the high grade tumours without formal measurement and that this would be of value in the grading of these tumours at an early stage of their investigation.

Our results have shown a good correlation between 
our modified Bloom and Richardson scores and the fine needle aspiration scores obtained and they have also shown that this is a reliable method for the separation of low grade ductal breast carcinomas from middle and high grade tumours. We failed to distinguish histological grades II and III ductal carcinomas using the preoperative cytology, however, perhaps reflecting the narrowness of these two histological grades.

Examination of the scores obtained for the separate variables assessed in the analysis of the ductal carcinoma smears has shown a good separation of nuclear size among the three histological grades and a gradation of scores for cell to cell cohesion and nuclear pleomorphism in the smears from the lowest to highest grade of carcinoma. These two latter variables, however, did not permit the reliable separation of the different histological grades on their own, and this underlines the need to assess multiple characteristics in smears. In view of the fact that nucleolar prominence seemed to be evenly distributed among the three histological grades we propose that this feature is omitted from future scoring systems.

We were unable to apply our scoring system to the group of fibreoadenomas in a way that permitted valid comparison with the scores obtained from the other lesions assessed. We felt unable to apply our system to the clustered epithelial component of the presumed fibroadenoma smears, ignoring those cells in the areas that intervene between the clusters. To do this would have entailed making a diagnostic assumption which we felt would have been invalid. It is our experience that the so called "classic" fibroadenoma pattern seen in breast aspirates is not unique to this group of lesions and that similar appearances may be seen in other benign breast diseases, particularly sclerosing adenosis and radial scar. What is more, smears from low grade carcinomas may give the initial impression of being composed of tight clusters of cohesive, small, epithelial cells and it only becomes apparent on closer scrutiny of these smears that cells intervening between the clusters are of the same epithelial type and not the "naked nuclei" of the fibroadenoma smear. We appreciate that the pleomorphism seen in aspirates from fibroadenomas often gives rise to concern among pathologists experienced in the examination of breast fine needle aspirates and that the application of a modified scoring system to this group of lesions may help to define the range of fine needle aspiration appearances that can be seen in histologically confirmed benign lesions. We emphasise the importance of assessing smears in the context of clinical and mammographic data.'

Our results have highlighted two particular areas of difficulty in the diagnostic assessment of breast fine needle aspirates:
1 There is a considerable degree of overlap of scores between our group of benign conditions and the low grade carcinomas. Clearly, no scoring system can take into account the complexity of appearances that are assessed in the evaluation of an aspirate to reach a diagnosis, but this does emphasise the need for caution when interpreting this diagnostically difficult group of aspirates. It also underlines the necessity for critical evaluation of all the material available for cytological assessment in conjunction with clinical and mammographic data.

2 We were unable to distinguish on cytological grounds ductal carcinomas from lobular carcinomas. It is accepted that histological grade is of considerable prognostic importance in ductal carcinoma of breast and that overall crude survival figures for ductal and lobular carcinoma are similar. We believe that this system, or a refined version of it, can identify reliably medium and high grade carciomas preoperatively and may therefore be of value in the subsequent management of this more aggressive form of disease. In view of the fact that our small number of lobular carcinomas showed the same spectrum of severity of cytological abnormality as seen in the ductal carcinomas we suspect that this system could be of considerable value in the grading of lobular carcinoma where no satisfactory histological system is yet available.

We anticipate that this system all allow a more critical evaluation of fine needle aspirates from breast lesions and may allow refinement of preoperative surgical management, possibly enabling serial studies of the response of breast carcinomas to such therapeutic measures as hormonal manipulation or chemotherapy when surgery is not indicated.

We are extremely grateful to Professor $\mathbf{R} \mathbf{N}$ MacSween for his guidance throughout the study. We thank Mr David Smellie and the technical staff of the cytology department, Western Infirmary, for their enthusiasm in assisting us with this study. We are grateful to Ms Sheila Pattison of the department of medical illustration, Western Infirmary, Glasgow, for her assistance with the preparation of the illustrations and Messrs Peter Kerrigan and David McComb for their photographic expertise.

This work has been presented in part to The Pathological Society of Great Britain and Ireland, St Bartholomew's Hospital, London, January 1988.

\section{References}

1 Bloom HJG, Richardson WW. Histological grading and prognosis in breast cancer. Br J Cancer 1957;11:359-77. 
2 Baak JPA, Kurver PHJ, De Shoo-Niewlaat, et al. Prognostic indications in breast cancer-morphometric methods. Histopathology 1982;6:327-39.

3 Walgren A, Silverswärd C, Zajicek J. Evaluation of noedle aspirates and tissue sections as prognostic factors in mammary carcinoma. Acta Cytol 1976;20:313-18.

4 Zajdela A, De La Riva LS, Ghossein NA. The relation of prognosis to nuclear diameter of breast carcinoma cells obtained by cytological aspiration. Acta Cytol 1979;23:75-80.

5 Kuenen-Boumeester V, Hop WJC, Blonk DI, Boon M. Prognostic scoring using cytomorphometry and lymph node status of patients with breast carcinoma. Eur J Cancer Clin Oncol 1984;20:337-45.

6 Mossler JA, McCarty KS, Woodward BH, Mitchener LM, Johnston WW. Correlation of mean nuclear area with oestrogen receptor content in aspiration cytology of breast carcinoma.
Acta Cytol 1982;26:417-21.

7 Cornelisse CJ, de Konig HR, Arentz PW, Raatgever JW, van Heerde P. Quantitative analysis of the nuclear area variation in benign and malignant breast disease. Anal Quant Cytol 1981;3:128-34.

8 Mouriquand J, Gozlan-Fior M, Villemain D, et al. Value of cytoprognostic classification in breast carcinomas. J Clin Pathol 1986;39:489-96.

9 Lamb J, Anderson TJ, Dixon MJ, Levack PA. Role of fine needle aspiration cytology in breast cancer screening. $J$ Clin Pathol 1987;40:705-9.

Requests for reprints to: Dr J St J Thomas, Department of Pathology, Western General Hospital, Crewe Road, Edinburgh EH4 2XU, Scotland. 